\title{
Análisis de Cobertura Urbana de los Nodos de Actividad Primaria Mediante un Estudio de Accesibilidad Territorial en Quibdó (Colombia)
}

\author{
Diego A. Escobar(1), Carlos F. Urazán ${ }^{(2)}$ y Carlos A. Moncada(3) \\ (1) Facultad de Ingeniería y Arquitectura, Universidad Nacional de Colombia - Sede Manizales. (e-mail: \\ daescobarga@unal.edu.co) \\ (2) Facultad de Ingeniería Civil, Universidad de la Salle, Bogotá (e-mail: caurazan@unisallle.edu.co) \\ (3) Facultad de Ingeniería, Universidad Nacional de Colombia - Sede Bogotá. (e-mail: \\ camoncadaa@unal.edu.co)
}

Recibido Sep. 26, 2016; Aceptado Nov. 25, 2016; Versión final Dic. 27, 2016, Publicado Oct. 2017

\begin{abstract}
Resumen
La presente investigación aplica técnicas geoestadísticas para explicar el nivel de cobertura que presenta la infraestructura de transporte de la ciudad de Quibdó en relación a los Nodos de Actividad Primaria (NAP), los cuales son aquellos que permiten suplir las necesidades diarias mínimas de una población: Salud, Educación, Universitarios y Seguridad. La metodología se basa en un análisis de accesibilidad a estos nodos mediante el cálculo de la Accesibilidad Media Integral que ofrece la red de infraestructura. El objetivo es analizar la ubicación de los NAP a partir de su relación con las características operativas de la red de infraestructura del transporte. Se obtuvo que los NAP que mejores características de cobertura espacial poseen son Educativos, seguidos por los NAP Salud y Universitarios, los cuales reportan niveles de cobertura semejantes de las variables población, número de viviendas y área; mientras que los nodos que reportan una cobertura más deficiente son los de Seguridad.
\end{abstract}

Palabras clave: accesibilidad; análisis de redes; cobertura; equipamientos; infraestructura de transporte

\section{Urban Coverage Analysis of the Primary Activity Nodes by use of a Territorial Accessibility Study in Quibdó (Colombia)}

\begin{abstract}
This research applies geostatistical techniques to explain the level of coverage presented by the transport infrastructure of the city of Quibdó in relation to the Primary Activity Nodes (PAN), which are those that allow meeting the minimum daily needs of a population: Health, Education, University studies and Security. The methodology is based on an analysis of accessibility to these nodes through the calculation of the Integral Mean Accessibility offered by the infrastructure network. The objective is to analyze the location of the PANs based on their relationship with the operational characteristics of the transport infrastructure network. It was obtained that the PANs that have the best spatial coverage characteristics are Educational, followed by the PAN Health and PAN Universities, which report similar levels of coverage of the variables population, number of households and area. While those nodes that report a poorer coverage are those of Security.
\end{abstract}

Keywords: accessibility; network analyst; coverage; equipment; transport infrastructure 


\section{INTRODUCCIÓN}

Quibdó, capital del Departamento del Chocó se ubica en un punto central de la región Pacifica Colombiana (Ver Figura 1). Se encuentra a orillas del rio Atrato a tan sólo $43 \mathrm{~m}$ sobre el nivel del mar con una temperatura promedio de $28^{\circ} \mathrm{C}$ y una topografía plana. Con una población aproximada de 107.140 habitantes en la zona urbana, es una ciudad considerada universitaria, en donde gran parte de la población tiene como actividad principal la realización de estudios superiores en diferentes instituciones que prestan dicho servicio. Actualmente la ciudad refiere un caos total en términos de planificación urbana, situación que se refleja en el poco desarrollo de las redes de soporte territorial, las cuales absorben desplazamientos de la periferia al centro, típicos de una ciudad monocéntrica. La red de infraestructura del transporte asciende aproximadamente a $197 \mathrm{~km}$ en su área urbana, de los cuales $25 \%$ son pavimentados, lo cual, combinado con que un $78 \%$ de los desplazamientos diarios se realizan en modos motorizados, ha aumentado considerablemente los índices de contaminación ambiental (Alcaldía de Quibdó, 2015). Uno de los mayores problemas urbanos es el uso del mototaxi como medio de transporte más popular, $42 \%$ del total de viajes diarios (Alcaldía de Quibdó, 2015), situación que se potencializa al encontrar que los principales nodos de actividad en la ciudad sólo son alcanzables en modos motorizados.
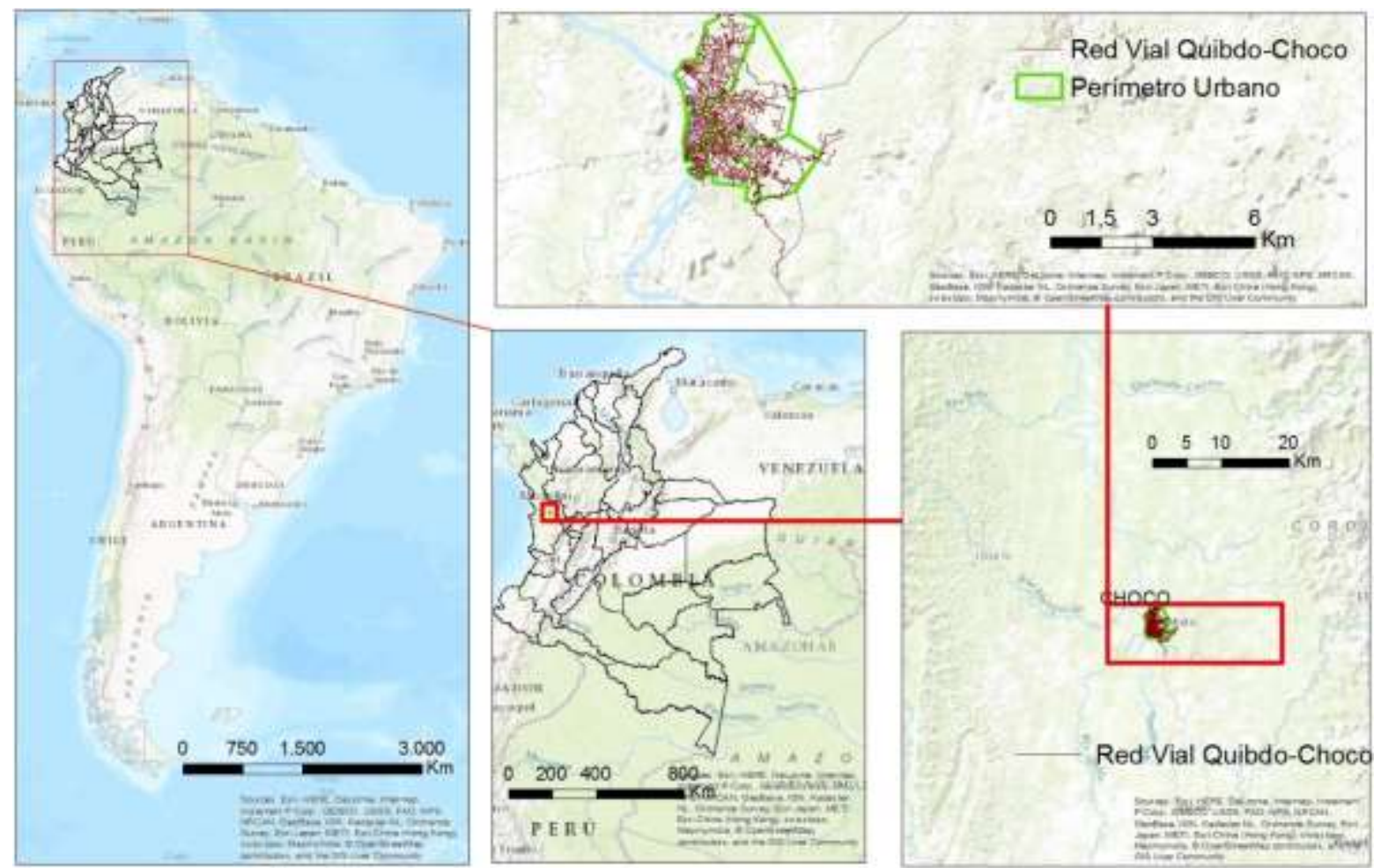

Fig. 1: Localización geográfica de la ciudad de Quibdó, Colombia

El desarrollo urbano sostenible de una ciudad está basado en diferentes aspectos, como la estructura urbana, el uso del suelo y el comportamiento de la movilidad o infraestructura del transporte. Estrategias de distribución espacial o buenas políticas de gestión del transporte no son suficientes para llegar al desarrollo deseado, en cambio se deben aplicar estrategias de desarrollo que promuevan estas bases como un conjunto (Hasibuna et al., 2014). La distribución espacial de áreas residenciales, de trabajo y de comercio (uso del suelo) contribuye a la generación de patrones de viaje condicionando las distancias y modos de viaje y, a su vez, afectando los consumos de energía y emisiones de contaminantes. Dentro de estos usos del suelo se deben enmarcar los denominados, Nodos de Actividad Primaria - NAP (Escobar et al., 2015a) cuyo principal fin es suplir, como mínimo, las principales necesidades de la población como lo son la educación (jardines, escuelas, colegios y universidades), salud y seguridad. Los NAP son importantes en las ciudades debido a la necesidad diaria de la población para acceder a estas actividades de manera rápida, siendo un papel fundamental la planeación de la localización de este tipo de nodos y la construcción de infraestructura del transporte que permita llegar a los mismos. De aquí que el termino accesibilidad se vea íntimamente relacionado con el desarrollo del suelo (Hansen, 1959).

El termino accesibilidad viene siendo usado desde comienzos del siglo XX en teorías de localización y planeamiento económico regional (Batty, 2009), tornándose más importante con la aparición del planeamiento en el transporte asociando la accesibilidad ofrecida por las redes de transporte y su relación con los patrones de viaje (Mitchell y Rapkin, 1954). Una definición concreta del concepto se ha planteado con la frase "la 
cercanía o proximidad entre un lugar y otro" (Tsou et al., 2005); otros investigadores complejizan la definición del concepto al manejarlo tanto ámbitos personales como medio ambientales (Iwarsson y Ståhl, 2003). Otra definición del concepto la define como la facilidad con que se puede llegar a un uso del suelo determinado desde cualquier ubicación usando un sistema de transporte específico (Dalvi, 1978; Koenig, 1980). Son pocos los departamentos de planeación urbana, de municipios en Colombia, que actualmente se enfocan en evaluar los efectos que sobre la movilidad y el transporte generan los usos del suelo, entendiendo que esta clase de efectos son bastante significativos (Waddell, 2002), así como que los viajes que se registran afectan las actividades y éstas a su vez afectan los patrones de desplazamientos de las personas (De Vos y Witlox, 2013). Es necesario entonces ofrecer un sistema de transporte que combine el adecuado acceso a las actividades (bienes y servicios) y usos del suelo, lo cual debe realizarse de forma medioambientalmente sostenible con el fin de no poner en peligro las oportunidades de las generaciones futuras (Black et al., 2002).

Una de las filosofías de movilidad que actualmente se encuentra vigente, son la ejecución de infraestructuras mediante la metodología de desarrollo orientado al transporte (Cervero y Kockelman, 1997), las cuales buscan lograr, en términos generales, tres objetivos: a) reducción de viajes motorizados, b) incremento de viajes en modos no motorizados, c) reducción de las distancias de viajes motorizados; lo anterior se lograría al crearse ambientes que impulsen el uso del transporte público y mejoren las características de accesibilidad y movilidad, mediante la promoción de conexiones multimodales (Handayeni, 2014), para lo cual debe contarse con mezcla de usos del suelo y una estación de transporte público en un área de alta densidad que permita la generación de adecuados espacios urbanos (Handayeni, 2014; Hasibuna et al., 2014). Por su parte, las medidas de accesibilidad e índices de cobertura de cualquier nodo de actividad, depende tanto de variables de percepción como de variables medibles (Morris et al., 1979), como el número de NAP, su ubicación, la eficiencia del transporte para llegar a éstos y la distribución de la población en relación con los NAP (Burkey, 2012). Dado lo anterior, la tendencia es la de implementar medidas para que los viajes que se realizan de forma más frecuente y que son más cortos, se realicen en modos no motorizados, dentro de áreas que mezclen usos del suelo (Cervero y Kockelman, 1997), para lo cual es necesario realizar análisis investigativos como el que en este caso se presenta.

Los análisis de accesibilidad poseen cuatro componentes principales: uso del suelo, transporte, temporalidad e individualidad; a partir del estudio de dichos componentes es posible definir cuatro tipos diferentes de estudios de accesibilidad: infraestructura, localización de actividades, medidas de carácter individual y beneficios económicos; así mismo, existen criterios teóricos, de operatividad, de comunicación, de evaluación social y evaluación económica (Geurs y Van We, 2004). Es importante comprender la accesibilidad como una necesidad primaria (Halden, 2011) no percibida, ya que por medio de ésta se pueden explicar las posibilidades existentes para llegar a diversas actividades como el empleo, conociéndose análisis en Holanda (Geurs y Ritsema, 2003), Alemania (Patuelli, et al., 2007; Reggiani, et al., 2010; Reggiani, et al., 2011), entre otros; así mismo, a nivel urbano, se conocen algunas investigaciones que se han ejecutado con el fin de conocer la cobertura que ofrecen algunos NAP, de forma agregada o por separado, como los nodos de actividad salud (Oppong y Hodgson, 1994; Tanser et al., 2006; Xia et al., 2008; Muñoz y Kallestal, 2012) y educativos (Pacione, 1989; Apparicio y Séguin, 2006; Foley y Pang, 2006; Younes et al., 2016). Vale la pena resaltar que el concepto se ha aplicado en variados campos de estudio, como: desarrollo económico regional (Rietveld y Nijkamp, 1993; Vickerman et al., 1999; Mackinnion et al., 2008), demografía (Kotavaara, 2011), cohesión social (López et al., 2008), sostenibilidad (Vega, 2011; Escobar et al., 2015a), turismo (Kastenholz et al., 2012), contaminación (Escobar et al., 2016), localización de servicios (Park, 2012; Higgs, 2013;), operatividad de modos de transporte (Botero et al., 2011; Escobar et al., 2012, Escobar et al., 2015b), entre otras.

Este tipo de estudios permiten realizar un análisis relacional entre la infraestructura vial y las líneas de deseo de movilidad de las comunidades, buscando la combinación más adecuada entre usos del suelo, estrategias de transporte y desarrollo urbano (Geurs y Van Wee, 2004); no obstante, esta investigación en particular, la accesibilidad se estudia en base a los criterios de operatividad y comunicación (Geurs y Van We, 2004), ya que se analiza la ubicación de los NAP en la ciudad de Quibdó, a partir de su relación con las características operativas de la red de infraestructura del transporte permitiendo conocer las posibilidades que tiene la población para alcanzar alguna de las actividades primarias en determinados rangos de tiempo medio de viaje, conectando la demanda y oferta de actividades (Halden et al., 2005), obteniéndose las características de cobertura geoespacial a los NAP en relación con las variables población, número de viviendas y área de la zona urbana estudiada. Desde el punto de vista de análisis de datos, se decidió el aplicar medidas de contorno (Geurs y Van We, 2004) dados el tipo y cantidad de la base de datos usada para los cálculos, lo cual permitió una interpretabilidad más aproximada de los resultados gráficos.

Es de resaltar que los países desarrollados y los que se encuentran en vías de desarrollo, utilizan los Sistemas de Información Geográfica (SIG) en el planeamiento de infraestructura y proyectos de transporte (Liu et al., 2006), así mismo han sido soporte para determinar la ubicación de cierto tipo de servicios comunitarios (Park, 2012; Khan y Samadder, 2014), estudios que en algunos casos se han realizado soportados en algoritmos 
de hormigas y SIG (Liu et al., 2006). En relación con el concepto de accesibilidad, se han creado herramientas SIG para su análisis en planeación urbana y de transporte (Liu y Zhu, 2004), estudios que relacionan usos del suelo y evaluación de impactos de transporte; así como modelos de Accesibilidad y su relación con análisis geográficos, todo lo anterior ha sido posible gracias a las nuevas capacidades de los SIG, tanto tecnológicas (Kwan y Weber, 2003) como de almacenamiento de información (Gómez y Barredo, 2005). Para esta investigación en particular, mediante la aplicación de geoestadística disponible en los software SIG, se calcularon curvas isócronas y la cobertura espacial según diferentes variables sociodemográficas, apoyados en la ejecución de los algoritmos (Zhang et al., 2009) de caminos mínimos desde los NAP a cualquier otro nodo de la red.

En los siguientes capítulos se aborda la metodología aplicada para la investigación, la formulación del modelo empleado, se discuten los principales resultados y se presentan las conclusiones.

\section{METOdOLOGÍA}

La metodología de investigación se compone de cuatro etapas principales (Ver Figura 2), a cada una de las cuales pertenecen subactividades que se deben llevar acabo para la ejecución de los cálculos. Las etapas de la metodología son: 1) Levantamiento de la red de infraestructura del transporte; 2) Georeferenciación de los NAP; 3) Aplicación del modelo geoestadístico; 4) Calculo de cobertura geoespacial.

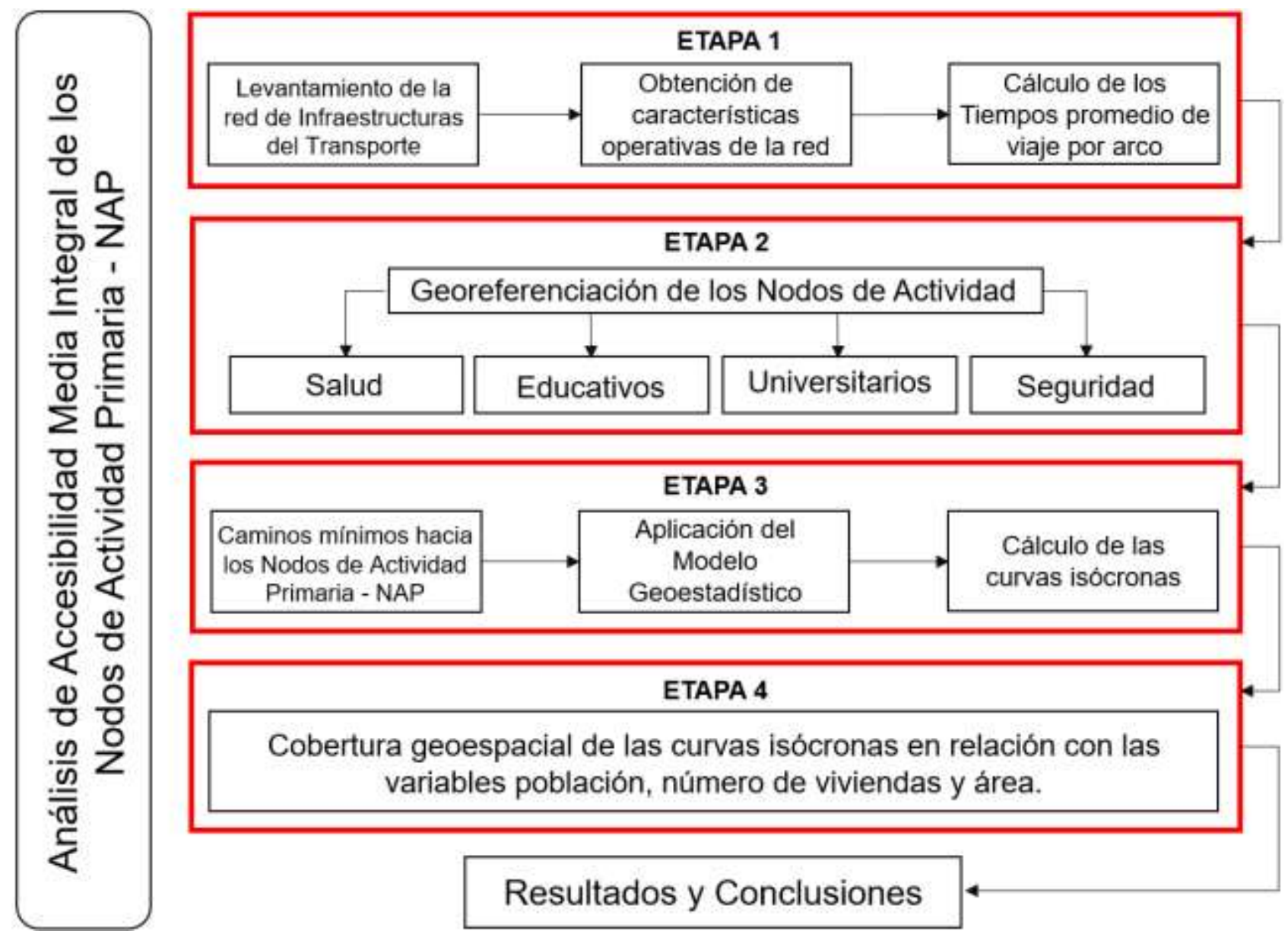

Fig. 2: Metodología aplicada

Etapa 1. Levantamiento de la red de infraestructura del transporte: Ésta consistió en el levantamiento de todas las características físicas y operativas de la red de infraestructura del transporte de la zona urbana del Municipio de Quibdó. Toda red se encuentra compuesta por la agregación de nodos (intersecciones) y arcos (segmentos de la vía) ubicados espacialmente. Para este caso en particular, la red analizada posee más de 2.700 nodos y 3.200 arcos; construida mediante el uso de aerofotografías y ortofotos que permitieron la validación completa de la red vial, así como la confirmación e identificación en campo de todos estos elementos. Para los cálculos, se tuvo en cuenta sólo la red de vehículo privado, dada la inexistencia de una red de transporte público o de una red multimodal de transporte como tal, y conociendo que más del $80 \%$ de los viajes motorizados se realizan en transporte privado (Alcaldía de Quibdó, 2015); por ello, se instalaron equipos GPS en vehículos particulares, con el fin de obtener datos sobre características operativas de la red 
de infraestructura del transporte. Es de resaltar que las velocidades de operación tenidas en cuenta para esta investigación son el promedio de las encontradas para cada arco de la red en vehículo privado y en un momento dado, es decir, no se encuentran ligados a una hora del día específica, entendiendo que ésta variable es un componente clave para los cálculos de accesibilidad (Geurs y Ritsema, 2001), así mismo, se resalta que otros investigadores han aplicado modelos de accesibilidad a redes multimodales de transporte, como en la investigación realizada en Finlandia por Kotavaara et al. (2011), y la realizada en España por López et al. (2008), en ambas se analizó la red multimodal conformada por la red de carreteras y la red ferroviaria.

Etapa 2. Georeferenciación de los NAP: A partir de documentos oficiales como el Plan de Ordenamiento Territorial (POT), el Plan de Desarrollo Municipal, el trabajo en campo y con ayuda del aplicativo Street View de Google Maps, se lograron ubicar dentro de la red los diferentes NAP a estudiar, a saber: a) NAP Salud, sitios en los que se encuentran servicios de asistencia médica o primeros auxilios; b) NAP Educativos, sitios en los que se imparte educación básica primaria y secundaria; c) NAP Universitarios, sitios en los que se imparte educación superior o técnica; d) NAP Seguridad, lugares a partir de los cuales se planifican todas la actividades relacionadas con la atención de emergencias y el control y gestión del orden público.

Etapa 3. Aplicación del Modelo Geoestadístico: Esta etapa es la relacionada con el cálculo de la Accesibilidad Media Integral ofrecida por la red de infraestructura de transporte actual y los cálculos de las curvas isócronas referenciadas con los NAP Salud, Educativos, Universitarios y Seguridad; la accesibilidad se analiza calculando el vector de tiempo medio de viaje (Tvi), el cual representa el tiempo mínimo que debe invertirse para alcanzar un NAP. Para los cálculos se usaron los módulos Network Analyst y Spatial Analyst del software ArcGis 10.2.1., a través de la aplicación de un algoritmo componente del SIG que permitió calcular la impedancia más baja (camino de tiempos mínimos) entre cada uno de los nodos de la red y los NAP. El vector de impedancias se relacionó con las coordenadas (longitud y latitud) de cada nodo de la red, generándose así la matriz a la cual se aplica el respectivo modelo geoestadístico con el fin de obtener las curvas isócronas de tiempo medio.

Etapa 4. Cálculo de cobertura geoespacial: El área urbana del municipio de Quibdó asciende a 19,9 km² (Alcaldía de Quibdó, 2015), alberga una población de 107.140 habitantes distribuidos en 28.932 viviendas, los datos demográficos usados en esta investigación son de origen censal, público y de alcance municipal, publicados por el Departamento Administrativo Nacional de Estadística - DANE. Mediante el uso de SIG y a partir de las curvas isócronas obtenidas, se calcularon los porcentajes de cobertura que cada una de éstas tienen en relación con las variables población, número de viviendas y área.

\section{FORMULACIÓN DEL MODELO}

Para el cálculo de la accesibilidad media integral desde cualquier nodo de la red hacia los NAP identificados en el área urbana del Municipio de Quibdó, se calculan los tiempos medios de viaje a partir de las velocidades de operación obtenidas a través de los GPS, estableciéndose ésta como parámetro base de los cálculos. El modelo que en esta investigación se formula, se soporta en el propuesto por Shen (2002), el cual se ha ajustado con fines de cobertura geoespacial de los NAP. Denotando $i \in I, i=\{1,2,3, \ldots\}$ como el conjunto de nodos de toda la red de infraestructura el transporte, $I$; igualmente, denotando $I \in L$ como los tipos de NAP a estudiar, $I=\{1,2,3,4\}$, salud, educativos, universitarios y seguridad. Estableciendo $j \in J$ como el número de nodos pertenecientes a cada grupo de NAP, $j=\{1,2,3, \ldots\}$. La variable de decisión $X_{i j}^{l}=1$ si el nodo de la red de infraestructura $i$ para un grupo de NAP / se conecta con algún NAP $j$. $D_{i j}^{l}=$ camino mínimo entre un nodo $i$ y un nodo $j$ en la red de transporte. $T^{\prime}=$ valor de la función objetivo. El objetivo es minimizar la función tiempo medio de viaje ( $T^{\prime}$ ) (Ver expresiones (1), (2) y (3)) desde los NAP conjuntos de nodos NAP (salud, educativos, universitarios y seguridad) hacia los demás nodos de la red de infraestructura del transporte. El modelo tiene como hipótesis que todos los NAP identificados pueden conectarse con cualquiera de los nodos haciendo uso de la red de infraestructura del transporte.

$$
T^{l}=\sum_{i} \sum_{j} D_{i j}^{l} X_{i j}^{l}
$$

De tal forma que,

$$
\begin{aligned}
& \sum_{i} \sum_{j} X_{i j}^{l}=j \text { para } l \in L \\
& \sum_{i} X_{i j}^{l}=1 \text {, para } \mathrm{j} \in \mathrm{J}, \mathrm{l} \in \mathrm{L}
\end{aligned}
$$


El cálculo de la Accesibilidad Media Integral es una medida de qué tan accesible, en términos de tiempo medio de viaje, es un sitio (nodo) o conjunto de sitios (NAP), en relación con la red de infraestructura del transporte; dicho cálculo permitirá establecer los sectores de la ciudad de Quibdó que refieren deficientes condiciones de acceso a un grupo de NAP en específico. Ésta se analizó a partir de la Expresión (4), en donde el vector Tvi representa el tiempo promedio de viaje desde cualquiera de los nodos del grafo hasta los nodos identificados como NAP. Ahora bien, con todos los arcos de la red de transportes cargados con la velocidad operativa y con la matriz de impedancias (distancias mínimas), se procede a calcular la matriz de tiempos medios mínimos de viaje. Donde, Tvi = tiempo de viaje mínimo promedio entre el nodo i y los demás nodos del grafo; $n=$ número total de nodos del grafo; $m$ = número de NAP de cada grupo.

$$
\overline{T_{v \iota}}=\frac{\sum_{j=1}^{m} t_{v i}}{(n-1)}, \text { siendo } i=1,2,3, \ldots, n ; j=1,2,3, \ldots, m \text {. }
$$

El modelo geoestadístico escogido para realizar la predicción de la variable de tiempo medio de viaje, fue el Kriging ordinario con semivariograma lineal, el cual se consideró como el más adecuado para el cálculo de los valores pronóstico dadas las características espaciales de los puntos a interpolar; así mismo, se decidió utilizar el semivariograma lineal dado que permitió la obtención de un mayor valor de validación cruzada que con un semivaruiograma esférico, exponencial o gaussiano, los cuales también fueron debidamente testeados. El modelo Kriging es un modelo geoestadístico basado en modelos de regresión y teorías de probabilidad, utilizado para la estimación de valores desconocidos a partir de datos de variables ya estimadas (tiempos medios de viaje desde y hacia nodos); con la asignación de pesos $\left(\mathrm{W}_{\mathrm{x}}\right)$ a los nodos que poseen tiempo de viaje, donde $W_{x}$ tiene un mayor valor en puntos observados cercanos a la ubicación que será extrapolada, es así que con el cálculo de un semivariograma de los valores de puntos conocidos y con relación al parámetro de ponderación de semivarianza media, se obtienen los valores para los puntos desconocidos. La ecuación fundamental y básica del método se observa en la Expresión (5). Donde $Z_{0}$ es el valor a estimar, $Z_{x}$ son los valores de los puntos conocidos, $W_{x}$ es el peso de los puntos conocidos respecto a los otros puntos del área geográfica a extrapolar y $\mathrm{S}$ son los números de puntos conocidos.

$$
Z_{0}=\sum_{i=1}^{S} Z_{x} w_{x}
$$

Para la semivarianza media se tiene que $\mathrm{h}$ es la distancia entre puntos, $\mathrm{n}$ son números pares de puntos que se definen según la distancia y z son los valores de puntos conocidos (Phatarapon et al., 2015) (Ver expresión (6)).

$$
f(h)=\frac{1}{2 n} \sum_{i=1}^{n}\left[z\left(x_{i}\right)-z\left(x_{i}+h\right)\right]^{2}
$$

Con la información demográfica y a través del aplicativo SIG, se calculó el porcentaje de población, número de viviendas y área, que se cubrían al relacionar dichas capas de información con las curvas isócronas obtenidas. La metodología que aplica en esta investigación, usa las velocidades promedio de los arcos, lo que se configura como un efecto simplificador en relación a la aplicación del modelo propuesto, ya que se está homogeniza el tiempo medio de viaje.

\section{RESULTADOS Y DISCUSIÓN}

Nodos de Actividad Primaria Salud: En la Figura 3 se observan las curvas de tiempos medios de viaje para evaluar la accesibilidad a los NAP Salud del municipio de Quibdó, el cual se encuentra cubierto por curvas isócronas entre 0,5 y 22,5 min. Se puede apreciar que la zona occidental del municipio se encuentra cubierta por curvas iguales o menores a $8 \mathrm{~min}$., indicando que desde dicho sector es posible alcanzar un NAP Salud si se invierte dicha cantidad de tiempo. Se identificaron un total de 52 nodos de este tipo de actividad, de los cuales 13 son de Ámbito Nacional, 15 de Ámbito Regional y 24 de Ámbito Local, la mayoría de ellos concentrados en el sector centro de la ciudad.

Los análisis de cobertura de las variables área, población y número de viviendas, permiten establecer conclusiones respecto a los sectores donde se concentra dicha actividad en relación con las características operativas de la red vial. En la figura 4a se observa la distribución de los porcentajes de cobertura de las variables población, número de viviendas y área que son cubiertas según las curvas isócronas obtenidas. Se observa que la mayor cobertura se obtiene para las variables población y número de viviendas con valores cercanos al $8 \%$ para tiempos medios de viaje de entre 5,5 y 6 minutos. Por otra parte, se observa que la variable área presenta un valor máximo de cobertura de 4,6\% correspondiente a un tiempo medio de viaje de entre 5,5 y 6 minutos. En la Figura 4b se observa la ojiva porcentual de las variables población, número de viviendas y área cubiertas por las curvas isócronas; es posible concluir que el $25 \%$ de las variables población y número de viviendas, logra alcanzar un NAP Salud si se invierten hasta 3,5 min. de tiempo medio de viaje, 
mientras que el $25 \%$ del área es cubierta por tiempos medios de viaje de hasta 6 min. La pendiente pronunciada que presentan las variables población y número de viviendas indica una buena accesibilidad hacia los NAP Salud del municipio, mientras que la baja pendiente de la curva en relación con la variable área, indica las características de baja densidad poblacional del municipio.

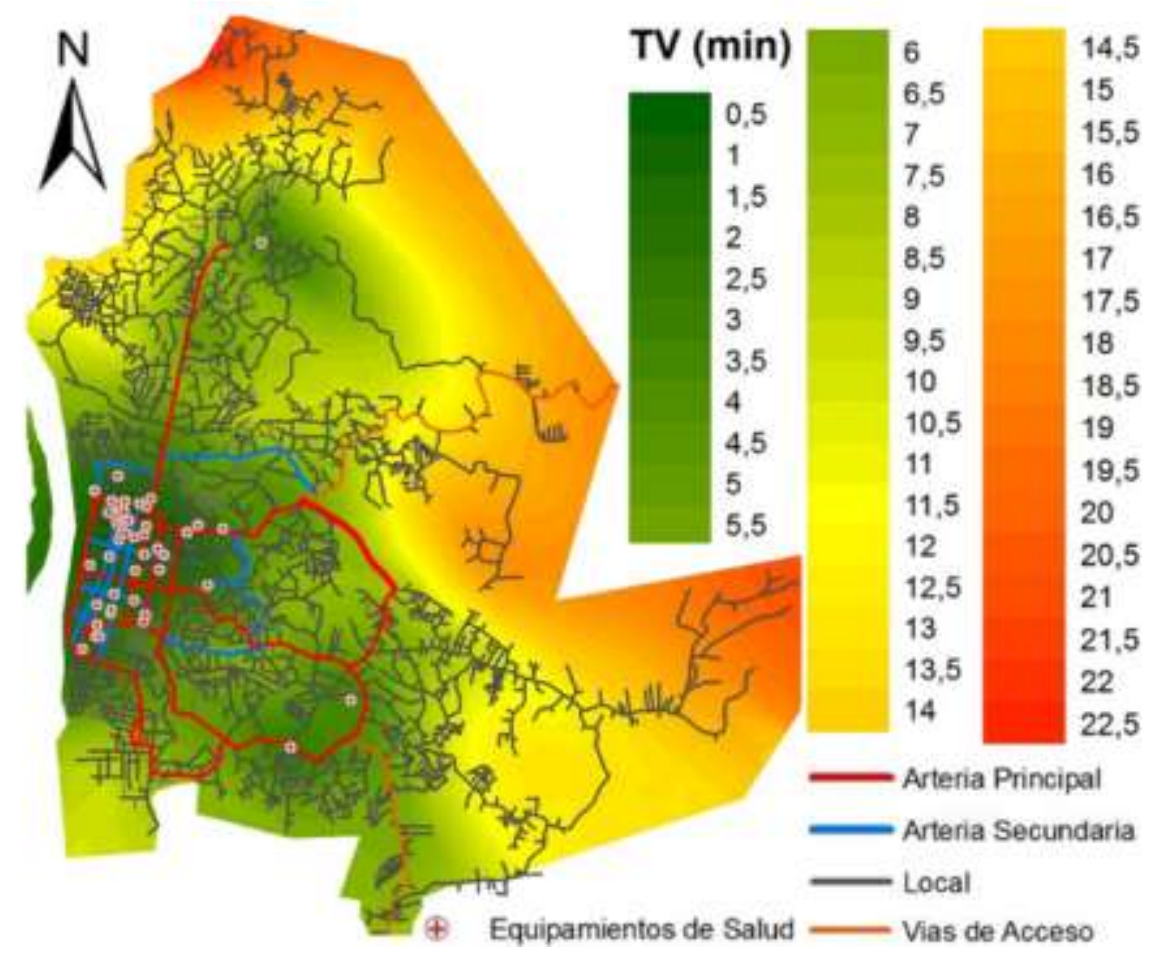

Fig. 3: Tiempos medios de viaje hacia los NAP Salud.

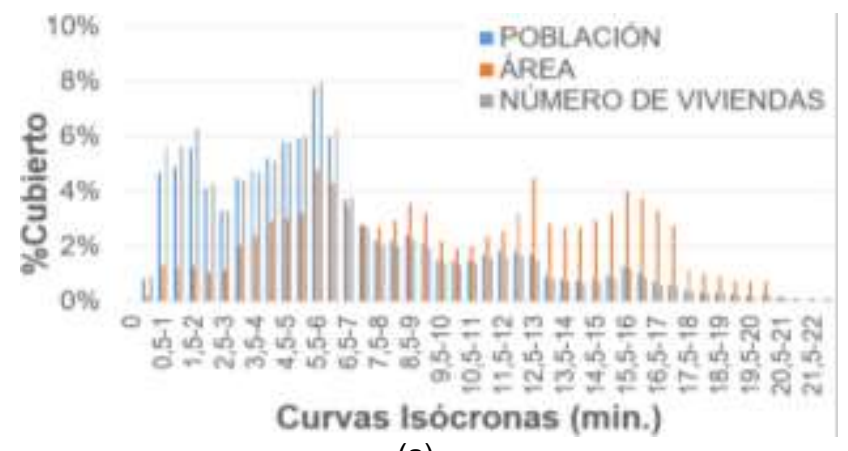

(a)

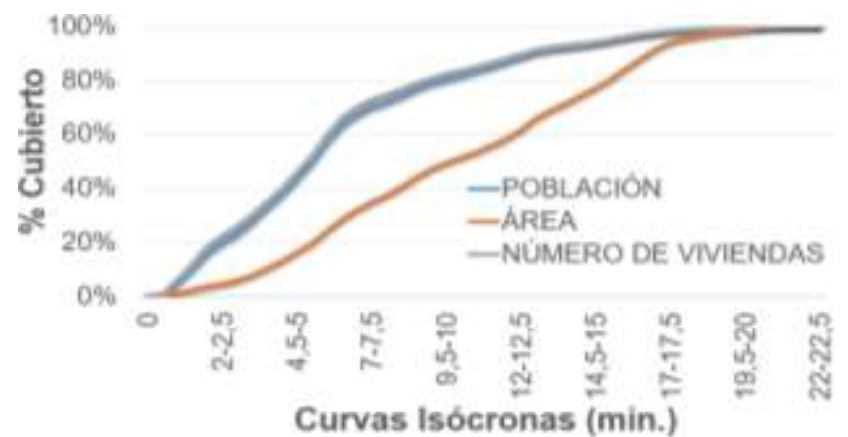

(b)

Fig. 4: a) Distribución porcentual de las variables población, número de viviendas y área cubiertas por las curvas isócronas para los NAP Salud. b) Ojiva porcentual de las variables población, número de viviendas y área cubiertas por las curvas isócronas para los NAP Salud.

Nodos de Actividad Primaria Educativos: En la Figura 5 se observan las curvas isócronas que permiten evaluar la accesibilidad ofrecida por la red de infraestructura del transporte hacia los NAP Educativos. Se observa que éstos se encuentran cubiertos por tiempos medios de viaje entre 1 y 20,5 min. De forma general se obtuvo que la zona occidental del municipio (sector centro de la ciudad) se encuentra cubierta por curvas isócronas de máximo 5 min., estas curvas se expanden a lo largo de los corredores principales hacia el norte y el oriente del municipio. Se identificaron un total de 31 NAP de este tipo, de los cuales 4 son de Ámbito Nacional, 2 de Ámbito Regional y 25 de Ámbito Local.

En la Figura 6a se observa la distribución de los porcentajes de cobertura de las variables población, número de viviendas y área cubiertas por las diferentes curvas isócronas. Se observa que la mayor cobertura se presenta para las variables población y número de viviendas con un $9,5 \%$ para las curvas isócronas que van de 3,5 a 4 min. Así mismo, se obtuvo que la variable área presenta un valor máximo de cobertura de 5,5\% correspondiente a un tiempo medio de viaje de entre 5 y 5,5 min. En la Figura $6 \mathrm{~b}$ se observa la ojiva porcentual para las variables población, número de viviendas y área cubiertas por las curvas isócronas. Se concluye que el $25 \%$ de la población y del número de viviendas logran acceder a un NAP Educativo si se invierten máximo 
2,5 min. de tiempo medio de viaje; de igual forma, para el $50 \%$ de cobertura de las mismas variables, se logran al invertir máximo 4 minutos de tiempo medio de viaje; en relación con la variable área, los valores correspondientes a los mismos porcentajes de cobertura, se obtienen tiempos medios de viaje casi el doble al obtenido para las otras variables analizadas.

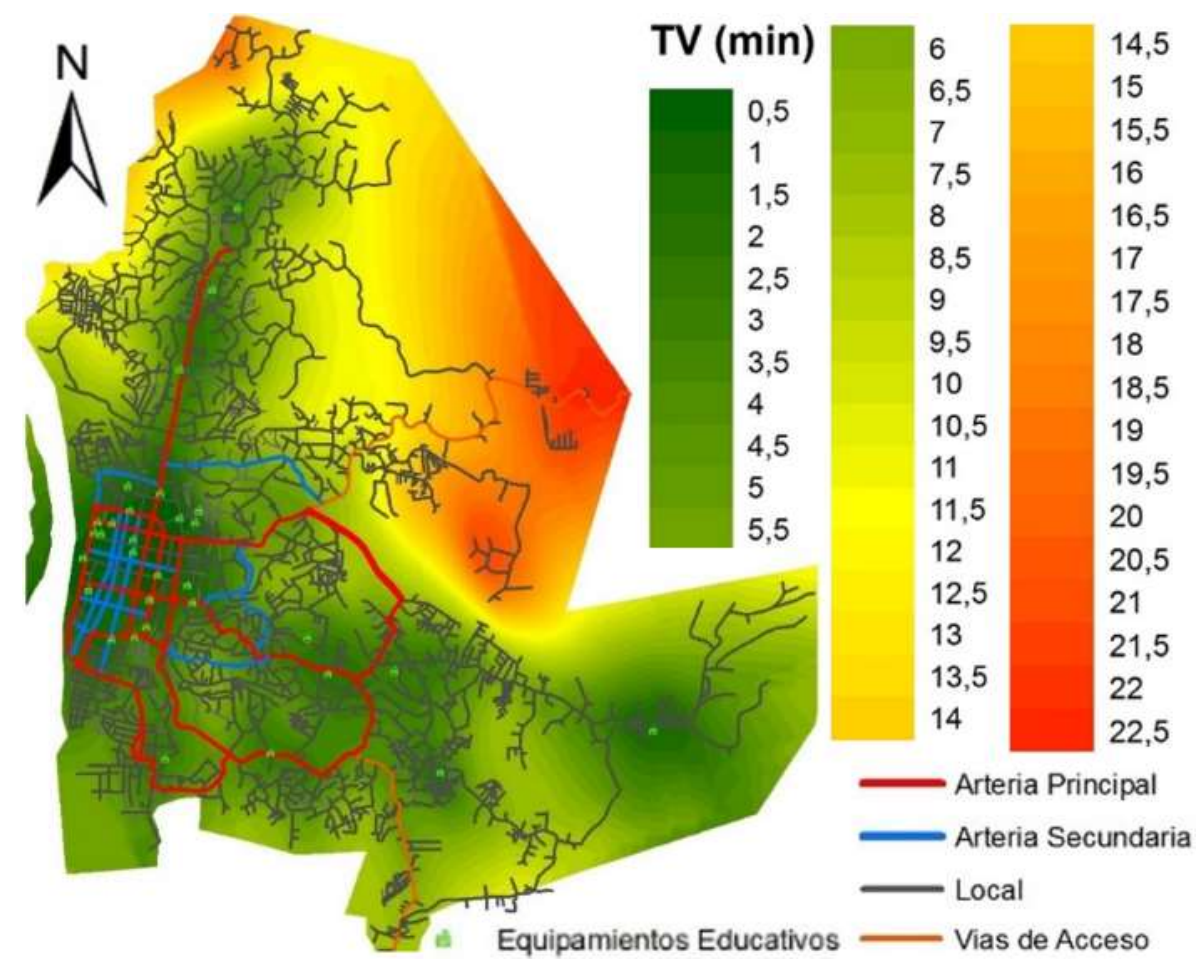

Fig. 5. Tiempos medios de viaje hacia los NAP Educativos.

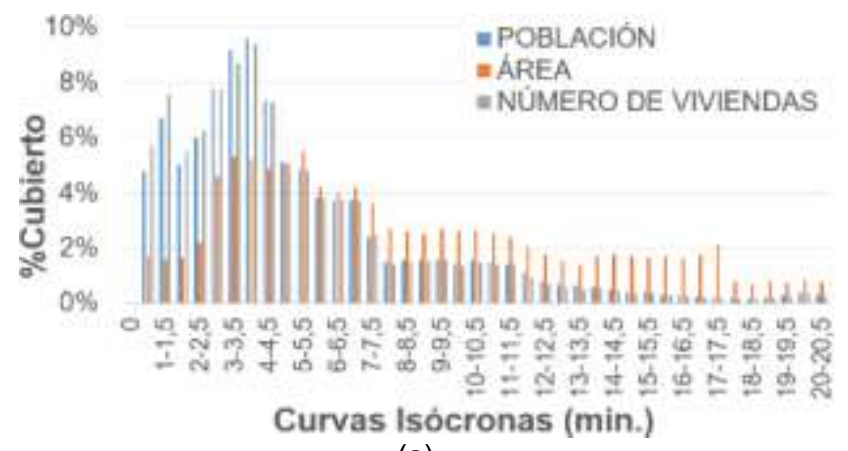

(a)

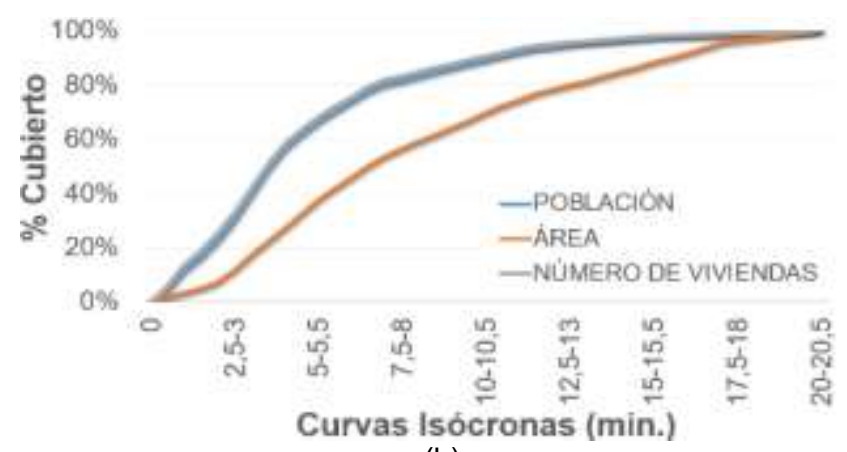

(b)

Fig. 6: a) Distribución porcentual de las variables población, número de viviendas y área cubiertas por las curvas isócronas para los NAP Educativos. b) Ojiva porcentual de las variables población, número de viviendas y área cubiertas por las curvas isócronas para los NAP Educativos.

Se realizó el análisis por separado para las instituciones de educación superior (Universidades), dada la importancia que éstas refiere para la población entendiendo que Quibdó es una de las ciudades en Colombia que tiene mayor número de estudiantes universitarios por cada 1000 habitantes, con un valor de 107 (MCV, 2016); en la Figura 7 se observan las curvas isócronas que permiten evaluar las características de accesibilidad a las universidades del municipio, se obtuvo que para este tipo de nodos de actividad existen curvas isócronas entre 1 y $26,5 \mathrm{~min}$. Se puede apreciar que nuevamente la zona occidental del municipio (sector centro de la ciudad) se encuentra cubierta por tiempos medios de viaje iguales o menores a 5 min. Se identificaron 9 NAP de este tipo, de los cuales 6 son de Ambito Nacional, 2 de Ámbito Regional y 1 de Ámbito Local.

En la Figura 8a se observa la distribución de los porcentajes de cobertura de las variables población, número de viviendas y área cubiertas, según las curvas isócronas obtenidas a dichos NAP. La mayor cobertura se presenta nuevamente para las variables población y número de viviendas con coberturas del 7,8\% y 8,8\%, respectivamente, para un tiempo medio de viaje de hasta $2,5 \mathrm{~min}$. En la Figura $8 \mathrm{~b}$ se observa la ojiva porcentual de cobertura de las variables población, número de viviendas y área cubiertas por las curvas 
isócronas. Se concluye que el $25 \%$ de las variables población y número de viviendas alcanzarían un NAP universitario si se invierten como mínimo $3 \mathrm{~min}$. de tiempo medio de viaje; mientras que el $25 \%$ del área es cubierta por tiempos medios de viaje de hasta 5,5 min. De igual forma es posible apreciar que el $50 \%$ de las variables población y número de viviendas se encuentran cubiertas por tiempos medios de viaje de mínimo 5,5 min.; mientras que el 50\% del área se alcanza a cubrir con un tiempo medio de viaje de 9 min. De nuevo se puede observar que la población y las viviendas tienen mejor cobertura por parte de este nodo de actividad, es decir, espacialmente la población está concentrada en ciertas áreas, generando la existencia de sectores del municipio que requieren mayores tiempos de viaje hasta las universidades.

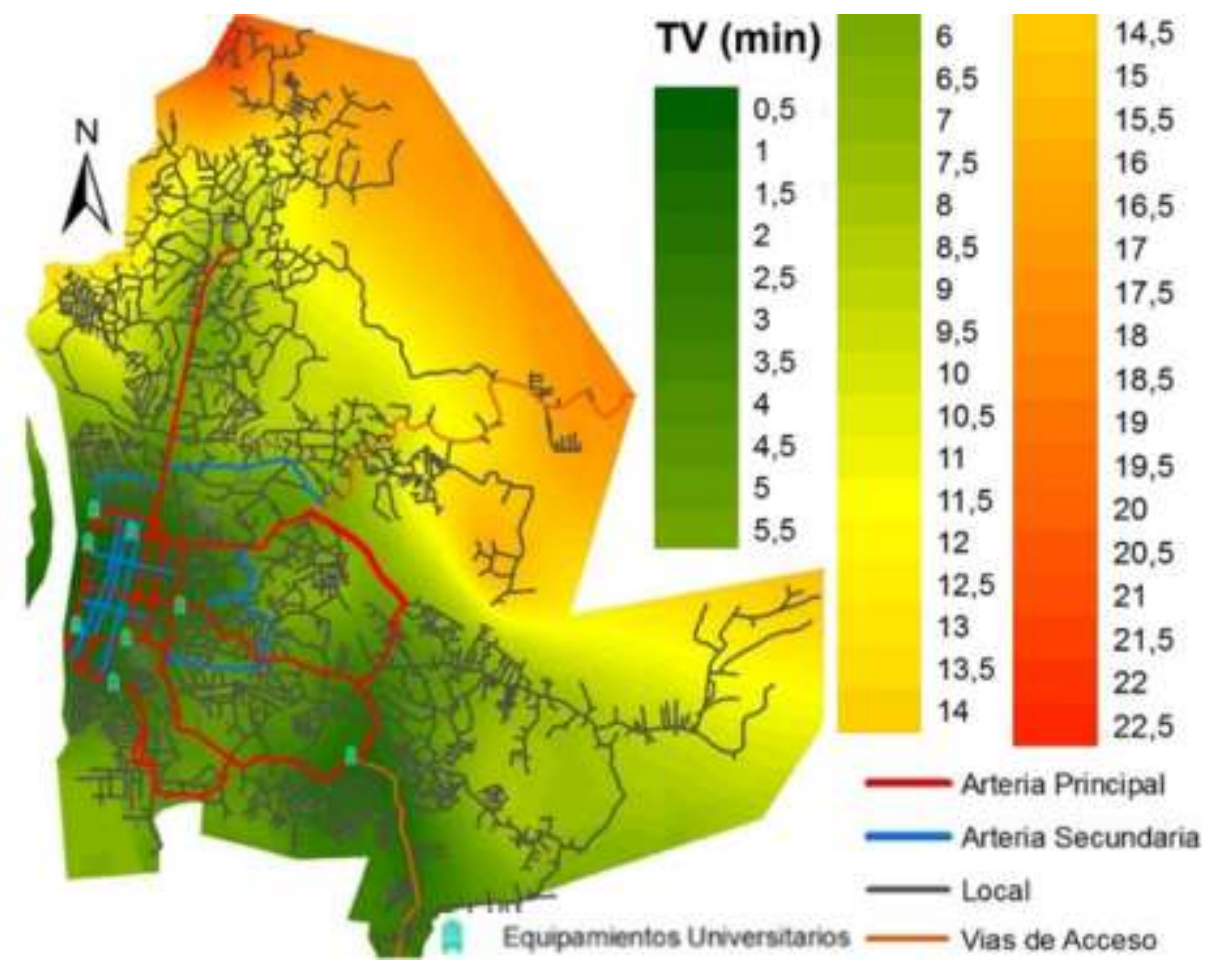

Fig. 7: Tiempos medios de viaje hacia los NAP Universitarios.

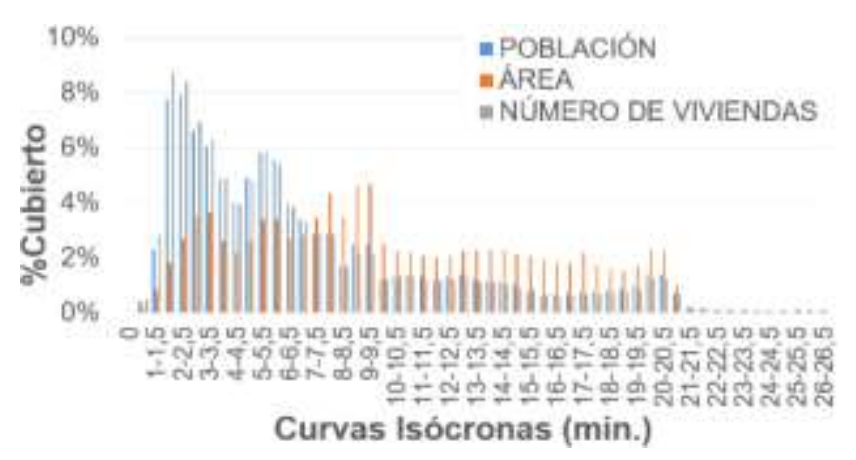

(a)

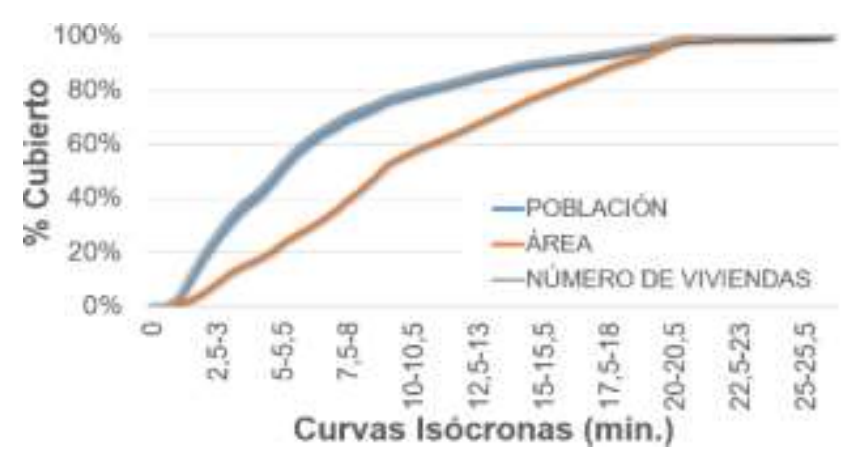

(b)

Fig. 8: a) Distribución porcentual de las variables población, número de viviendas y área cubiertas por las curvas isócronas para los NAP Universitarios. b) Ojiva porcentual de las variables población, número de viviendas y área cubiertas por las curvas isócronas para los NAP Universitarios.

Nodos de Actividad Primaria Seguridad: En la Figura 9 se observan las curvas isócronas que permiten evaluar la accesibilidad media integral ofrecida por la red de infraestructura del transporte en relación con los NAP Seguridad; se obtuvo que para alcanzar dichos nodos, se deben invertir tiempos medios de viaje entre 2 y 25 min. La zona céntrica del municipio (sector occidente) es la que se encuentra cubierta por tiempos medios de viaje menores, ya que la mayor cantidad de este tipo de nodos se encuentran ubicados en dicha zona. Se identificaron un total de 5 nodos de actividad de este tipo, de los cuales 2 son de Ámbito Nacional y 3 de Ámbito Regional.

En la Figura 10a se observa la distribución de los porcentajes de cobertura de las variables población, número de viviendas y área cubiertas, según las curvas isócron as obtenidas. Para tiempos medios de viaje de hasta 2,5 min., la variable número de viviendas se cubre hasta en un 7\%, mientras que la variable población llega tan solo a un $6 \%$ de cobertura. Por otra parte, analizando la variable área, se obtuvo que presenta un valor 
máximo de cobertura de $4 \%$ correspondiente a un tiempo medio de viaje de hasta 9,5 min. En la Figura 10b se observa la ojiva porcentual de cobertura de las variables población, número de viviendas y área cubiertas por las curvas isócronas; se concluye que el $25 \%$ del número de viviendas se cubre con un tiempo medio de viaje de hasta 4 min., mientras que para igual porcentaje de cobertura de las variables población y área, se deben invertir 4,5 y 8,5 min., respectivamente. De igual forma es posible apreciar que el $50 \%$ de la población y número de viviendas se podría cubrir si se invierten mínimo $8,5 \mathrm{~min}$. de tiempo medio de viaje, mientras que el 50\% del área se cubriría invirtiendo mínimo 13,5 min. de tiempo medio de viaje. Se puede apreciar de la misma manera aunque en menor proporción que lo observado en los otros nodos de actividad primaria que los nodos de seguridad presentan mejor cobertura para las variables población y número de viviendas para la variable área, lo cual se relaciona directamente con las características de densidad poblacional de la ciudad.

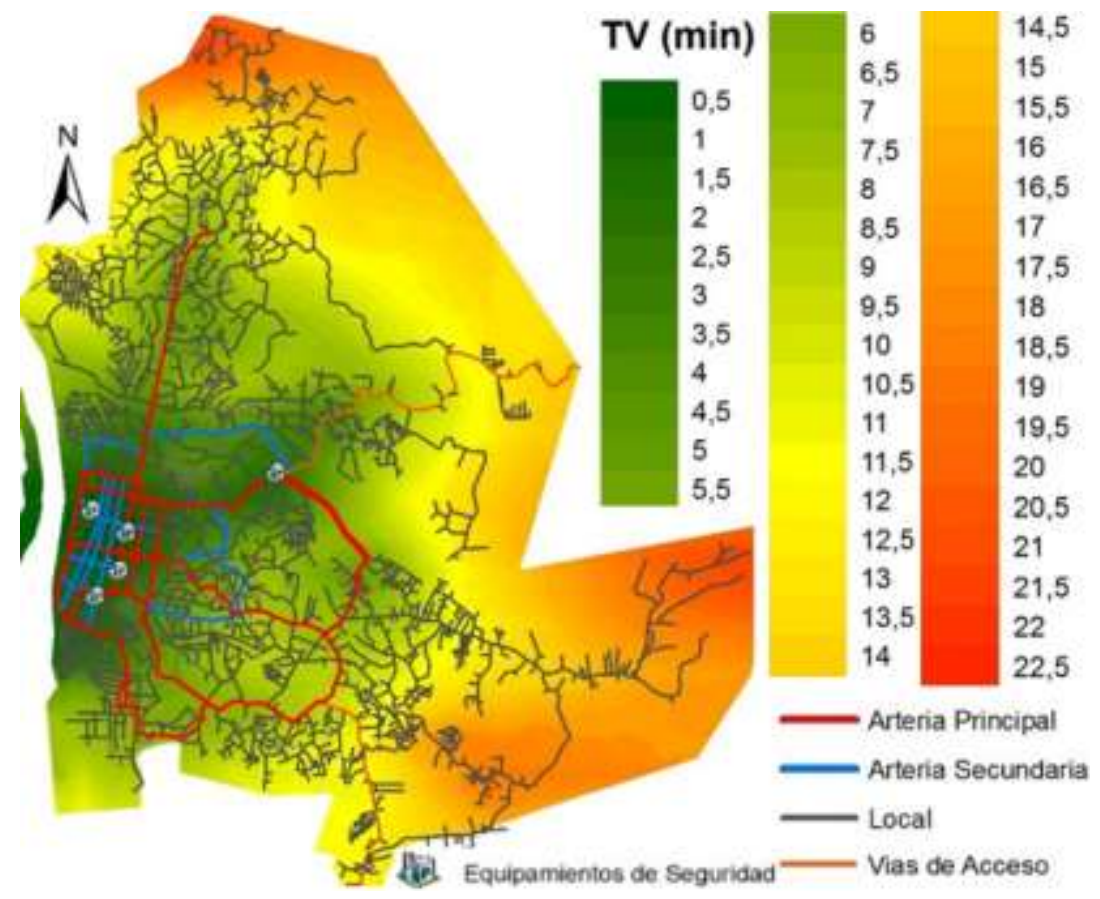

Fig. 9: Tiempos medios de viaje hacia los NAP Seguridad.

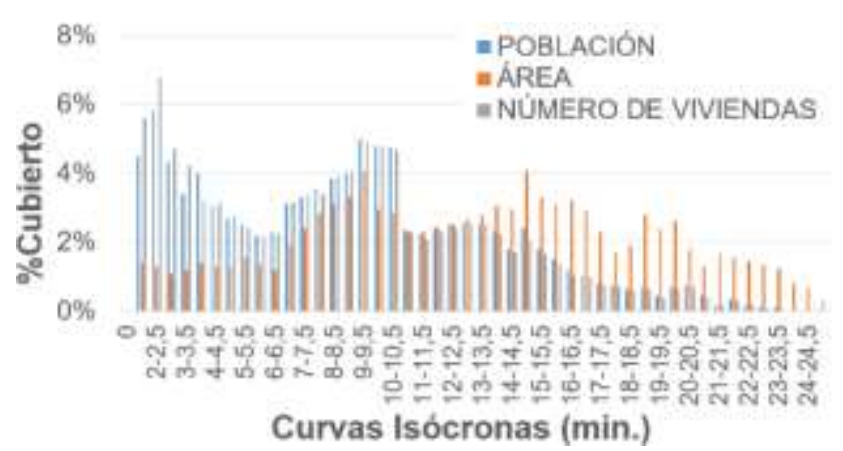

(a)

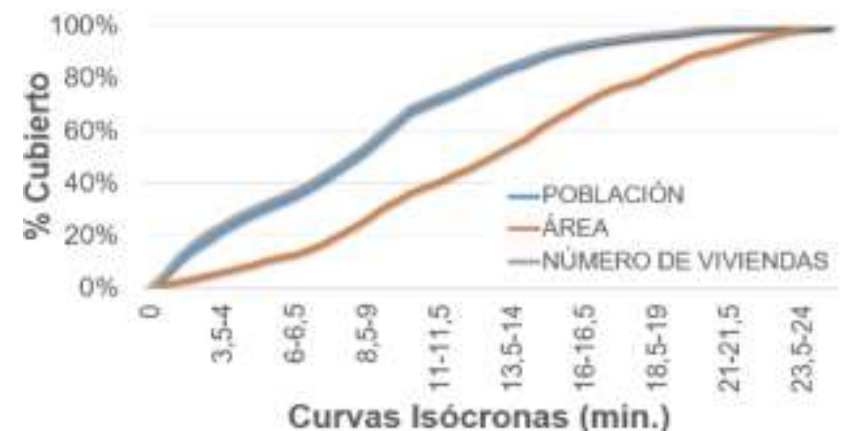

(b)

Fig. 10: a) Distribución porcentual de las variables población, número de viviendas y área cubiertas por las curvas isócronas para los NAP Seguridad. b) Ojiva porcentual de las variables población, número de viviendas y área cubiertas por las curvas isócronas para los NAP Seguridad.

En términos de movilidad, la situación del uso porcentual de vehículos automotores en la ciudad de Quibdó es más crítica que en Manizales, 78\% en la primera (Alcaldía de Quibdó, 2015) y 68\% en la segunda (Alcaldía de Manizales, 2011), teniendo que el uso del transporte público colectivo urbano es menor en Quibdó que en Manizales, así como el porcentaje de viajes en modos no motorizados; no obstante, es posible comparar los resultados de cobertura obtenidos en la ciudad de Quibdó con los obtenidos en la ciudad de Manizales, ya que en ambas se aplicó el mismo procedimiento metodológico en el marco de los Planes Integrales de Movilidad Urbana; así mismo, ambas ciudades son de tamaño medio y capitales de departamento ubicadas en la región occidente colombiana. Comparando los valores de cobertura de los NAP Educativos, se obtuvo que en la ciudad de Quibdó, el $62 \%$ de la población, dada las características operativas de la red de infraestructura del transporte, tiene la posibilidad de acceder a uno de ellos, lo que podría considerarse bajo si se comparase con el valor obtenido en Manizales, $72 \%$ para el mismo período de tiempo medio de viaje. 
Por otra parte, los NAP Universitarios en la ciudad de Quibdó registran una cobertura del $45 \%$ de la población en un tiempo medio de viaje de 5 min., mientras que en Manizales dicha cobertura llega a tan sólo el 15\% para igual periodo de tiempo (Escobar y García, 2012), lo cual es bastante diciente, ya que ambas ciudades refieren valores semejantes del número de estudiantes por cada mil habitantes, 107 y 112, respectivamente (MCV, 2016); así como son las dos ciudades en Colombia con mayor cantidad de universidades por habitante (Alcaldía de Manizales, 2011, Alcaldía de Quibdó, 2015). Los anteriores resultados apoyarían la promoción de modos de transporte más sostenibles para alcanzar este tipo de nodos y que claramente muestra la diferencia que las posibilidades topográficas proporcionan en una u otra ciudad, Quibdó plana y Manizales montañosa.

En relación con los NAP Salud, Manizales registra para un tiempo medio de viaje de 5 min. una cobertura del $40 \%$ de la población (Escobar et al., 2015), valor menor que el registrado en Quibdó (45\%), esto lleva a pensar que los NAP Salud en la ciudad de Quibdó se encuentran mejor ubicados en relación con la distribución geográfica de la población. Situación contraria se obtuvo en relación con los NAP Seguridad, en donde para el caso de Quibdó, el porcentaje de cobertura relacionado con un tiempo medio de viaje de 5 min. llega al $28 \%$, mientras que en Manizales dicho porcentaje es del $39 \%$.

\section{CONCLUSIONES}

En la Figura 11 se presenta una comparación de ojivas porcentuales de cobertura de la variable población para los NAP analizados. De forma general, se tiene que si una curva de cobertura se encuentra más hacia la derecha del origen y posee una menor pendiente, indicaría que para alcanzar dicho nodo de actividad se debe invertir un mayor tiempo medio de viaje. Se concluye que el NAP que mayor cobertura refiere sobre la población del municipio de Quibdó, es el Educativo, seguido en orden por los nodos Salud, Universitarios y Seguridad, respectivamente; indicando que en este municipio, en relación con las características de accesibilidad territorial urbana, es la actividad de prestación de seguridad la que más deficiencia posee en relación con su ubicación geoespacial y en relación con las características operativas de la red vial. Se concluye que en relación con los valores de cobertura obtenidos, al comparar con la ciudad de Manizales, los NAP Universitarios y Salud refieren mayor cobertura de población en menores tiempos medios de viaje, mientras que los NAP Educativos y Seguridad es contrario.

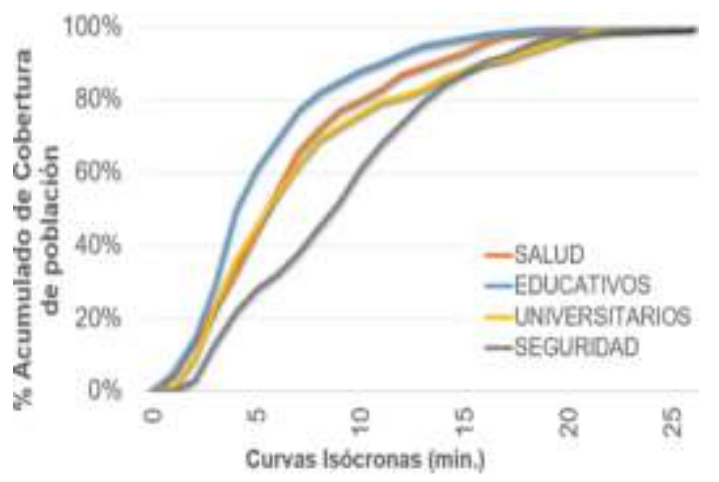

Fig. 11: Comparación del \% de cobertura de población para los NAP estudiados (Salud, Educativos, Universitarios y Seguridad).

Teniendo en cuenta las características topográficas de la ciudad de Quibdó (en su mayoría plana), así como que el $78 \%$ de los desplazamientos diarios se realizan en modos motorizados y que uno de los mayores problemas urbanos es el actual uso del mototaxi como medio de transporte más popular $(42 \%$ del total de viajes diarios), vale la pena apoyar de forma decidida la implementación de una red de ciclorutas municipal; es este un proyecto que se articula adecuadamente con la actual política de movilidad sustentable incluida en el Plan Integral de Movilidad de la ciudad (Alcaldía de Quibdó, 2015) y que dada la posición geoespacial de los NAP Educativos y Universidades, sería uno de los modos de transporte que mayor beneficio traería para la movilidad de la ciudad y mejora de la calidad de vida de sus habitantes. Se obtuvo que las peores condiciones de accesibilidad se presentan para los NAP Seguridad, lo cual es consecuencia tangible de una deficiencia en las características operativas de la red vial, lo cual, a su vez, se presenta por una inadecuada definición funcional de los modos de transporte que por ella discurren, así como de una errada planificación urbano-estratégica en relación con la ubicación de los NAP. A pesar de ello, es necesario reconocer que los NAP poseen lógicas locacionales diferentes, así como características de demanda de los servicios que los NAP prestan, los cuales varían a lo largo del día y de la semana. Se concluye que a través de los SIG es posible analizar y comprender con más detalle cómo la infraestructura de transporte y sus características operativas influyen directamente sobre las condiciones de accesibilidad presentes en un territorio, en relación con la ubicación de las actividades que en éste se desarrollan. 


\section{AGRADECIMIENTOS}

Los autores expresan su agradecimiento a los estudiantes pertenecientes a los semilleros de investigación en "Movilidad Sostenible" y "Planeación Urbana" de la Universidad Nacional de Colombia - Sede Manizales.

\section{REFERENCIAS}

Alcaldía de Manizales, Secretaría de Tránsito y Transporte, Plan Maestro de Movilidad de la ciudad de Manizales 2010 - 2040. Universidad Nacional de Colombia - Sede Manizales. Manizales (2011)

Alcaldía de Quibdó, Secretaría de Infraestructura, Plan Integral de Movilidad de la ciudad de Quibdó 2015 2045. Universidad Nacional de Colombia - Sede Manizales. Manizales (2015)

Apparicio, P. y Séguin, A., Measuring the accessibility of services and facilities for residents of public housing in Montreal, doi:10.1080/00420980500409334, Urban studies, 43(1),187-211 (2006)

Batty, M., Accessibility: in search of a unified theory, doi:10.1068/b3602ed, Environment and Planning B: Planning and Design, 36,191-194 (2009)

Black, J., Paez, A. y Suthanaya, P., Sustainable Urban Transportation: Performance Indicators and Some Analytical Approaches, doi:10.1061/(ASCE)0733-9488(2002)128:4(184), Journal of Urban Planning and Development, 128(4),184-209 (2002)

Burkey, M., Decomposing geographic accessibility into component parts: methods and an application to hospitals, doi:10.1007/s00168-010-0415-3, Annals of Regional Science, 48 (3),783 - 800 (2012)

Botero, R., Murillo, J. y Jaramillo, C., Decision strategies for evaluating pedestrian accessibility, https://goo.gl/BckBM8, Revista Dyna, 78 (168), 28-35 (2011)

Cervero, R. y Kockelman, K., Travel Demand And The 3ds: Density, Design And Diversity, doi:10.1016/S1361-9209(97)00009-6, Transportation Research Part D: Transport and Environment, 2(3), pp.199-219 (1997)

Dalvi, M., Behavioural modelling accessibility, mobility and need: concepts and measurement, in D. A. Hensher and P. R. Stopher, Eds.,Behavioural Travel Modelling, London, Croom Helm (1978)

De Vos, J. y Witlox, F., Transportation policy as spatial planning tool; Reducing urban sprawl by increasing travel costs and clustering infrastructure and public transportation, doi:10.1016/j.jtrangeo.2013.09.014, Journal of Transport Geography, 33, 117-125 (2013)

Escobar, D. y García, F., Análisis de Accesibilidad a nodos de actividad en Manizales (Colombia), Facultad de Ingeniería y Aquitectura. Universidad Nacional de Colombia. ISBN: 978-958-761-127-4. Manizales (2012)

Escobar, D., Cadena, C. y Salas, A., Cobertura Geoespacial de nodos de actividad primaria. Análisis de los aportes a la sotenibilidad urbana mediante un estudio de accesibilidad territorial, doi:10.14508/reia.2015.12.23.13-27, Revista EIA, 12(23),13-27 (2015a)

Escobar, D., Tapasco, O. y Giraldo, J., Medición de Desempeño del Sistema de Transporte Cable Aéreo de la Ciudad de Manizales en Colombia, usando Tres Enfoques: Analítico, Simulado y de Accesibilidad Urbana, doi:10.4067/S0718-07642015000600020. Inf. Tecnol. [online]. 26(6) 199-210 (2015b)

Escobar, D., Martínez, S. y Moncada, C., Relación entre PM10 y condiciones de Accesibilidad territorial urbana en Manizales (Colombia) 2012 - 2015, doi: 10.4067/S0718-07642016000600027. Inf. Tecnol. [online]. 27(6), 273-284 (2016)

Foley, R. y Pang, L., Alternative education programs: Program and student characteristics, The High School Journal, 89(3), 10-21 (2006)

Geurs, K. y Ritsema van Eck, J., Accessibility Measures: Review and Applications. RIVM Report 408505006. National Institute of Public Healthand the Environment, Bilthove (2001)

Geurs, K. y Ritsema van Eck, J., Evaluation of accessibility impacts of land-use scenarios: The implications of job competition, land-use, and infrastructure developments for the Netherlands, doi:10.1068/b12940, Environment and Planning B: Planning and Design, 30, 69-87 (2003) 
Geurs, K. y Van Wee, B., Accessibility evaluation of land-use and transportstrategies: review and research directions, doi:10.1016/j.jtrangeo.2003.10.005, Journal of Transport Geography, 12(2),127-140 (2004)

Gómez, M. y Barredo, J.I., Sistemas de información geográfica y evaluación multicriterio en la ordenación del territorio, $2^{a}$ Ed., 279, Ra-Ma Editorial, Madrid, España (2005)

Halden, D., The use and abuse of accessibility measures in UK passenger transport planning, doi:10.1016/j.rtbm.2011.05.001, Transportation Business \& Management, 2,12-19 (2011)

Halden, D., Jones, P. y Wixey, S., Accessibility Analysis Literature Review. Working Paper 3, disponible en: https://goo.gl/hQndXE (2005)

Handayeni, KDME, TOD Best Practice: Lesson Learned for GHG Mitigation on Transportation Sector in Surabaya City, Indonesia, doi:10.1016/j.sbspro.2014.07.340, Procedia-Social and Behavioral Sciences, 135, 152-158 (2014)

Hansen, W., How accessibility Shapes Land Use, J. of the American Institute of Planners, 25(2), 73-76 (1959)

Hasibuana, H., Soemardi, T., Koestoerb, R. y Moersidikc S., The Role of Transit Oriented Development in constructing urban environment sustainability, the case of Jabodetabek, Indonesia, doi: 10.1016/j.proenv.2014.03.075, Procedia Environmental Sciences, 20,622-631 (2014)

Higgs, G., Langford, M. y Fry, R., Investigating variations in the provision of digital services in public libraries using network-based GIS models, doi:10.1016/j.lisr.2012.09.002, Library \& Information Science Research, 35 (1), 24-32 (2013)

Iwarsson, S. y Ståhl, A., Accessibility, usability and universal design-positioning and definition of concepts describing person-environment relationships, doi:10.1080/dre.25.2.57.66, Disability \& Rehabilitation, 25(2), 57-66, (2003)

Kastenholz, E., Eusébio, C., Figueiredo, E. y Lima, J., Accessibility as Competitive Advantage of a Tourism Destination: The Case of Lousã, in Field Guide to Case Study Research in Tourism, Hospitality and Leisure (Advances in Culture, Tourism and Hospitality Research, Volume 6, K.F. Hyde, C. Ryan and A.G. Woodside (Ed.), doi: 10.1108/S1871-3173(2012)0000006023, Emerald Group Publishing Limited, 369-385 (2012)

Khan, D. y Samadder, S.R., Municipal solid waste management using Geographical Information System aided methods: A mini review, doi: 10.1177/0734242X14554644, Waste Management \& Research, 32(11),1049$1062(2014)$

Koenig, J., Indicators of urban accessibility: theory and application, doi:10.1007/BF00167128, Transportation, 9(2), 145-172 (1980)

Kotavaara, O., Antikainen, H. y Rusanen, J., Population change and accessibility by road and rail networks: GIS and statistical approach to Finland 1970-2007, doi:10.1016/j.jtrangeo.2010.10.013, Journal of Transport Geography, 19 (4), 926-935 (2011)

Kwan, M. y Weber, J., Individual Accessibility Revisited: Implications for Geographical Analysis in the Twentyfirst Century, doi: 10.1353/geo.2003.0015, Geographical Analysis 35(4), 341-353 (2003)

Liu, N., Huang, B. y Chandramoul, M., Optimal Siting of Fire Stations Using GIS and ANT Algorithm, doi: 10.1061/(ASCE)0887-3801(2006)20:5(361), Journal of Computing in Civil Engineering, 20(5), 361-369 (2006)

Liu, S. y Zhu, X., Accessibility Analyst: an integrated GIS tool for accessibilityanalysis in urban transportation planning, doi: 10.1068/b305, Environment and Planning B: Planning and Design, 31(1), 105-124 (2004)

López, E., Gutierrez, J. y Gómez, G., Measuring regional cohesion effects of large-scale transport infrastructure investment: an accessibility approach, doi: 10.1080/09654310701814629, European Planning Studies, 16 (2), 277-301 (2008)

Mackinnon, D., Pirie, G. y Gather, M., Transport and economic development. In R. Knowles, J. Shaw, \& I. Docherty, Editors, Transport Geographies: Mobilities, Flows and Spaces (10-28). Blackwell Publishers, Oxford (2008)

Manizales Como Vamos - MCV. Informe Técnico: "Cómo vamos en Educación Superior". Manizales, disponible en: https://goo.gl/FbWM5x (2016) 
Mitchell, R. y Rapkin, C., Urban Traffic: A Function of Land Use, Columbia University Press, New York (1954)

Morris, J., Dumble, P. y Wigan, M., Accessibility indicators in transport planning, doi:10.1016/01912607(79)90012-8, Transportation Research, A, 13, 91-109 (1979)

Munoz, U. y Kallestal, C., Geographical accessibility and spatial coverage modeling of the primary health care network in the Western Province of Rwanda, doi:10.1186/1476-072X-11-40, International Journal of Health Geographics, 11 (40) (2012)

Oppong, J. y Hodgson, M., Spatial accessibility to health care facilities in Suhum District, Ghana, doi: 10.1111/j.0033-0124.1994.00199.x, The Professional Geographer, 46(2),199-209 (1994)

Pacione, M., Access to urban services - the case of secondary schools in Glasgow, The Scottish Geographical Magazine, 105(1),12-18 (1989)

Park, S., Measuring public library accessibility: a case study using GIS, doi:10.1016/j.lisr.2011.07.007, Library \& Information Science Research, 34 (1), 13-21 (2012)

Patuelli, R., Reggiani, A., Gorman, S., Nijkamp, P. y Bade, F., Network analysis of commuting flows: a comparative static approach to German data, doi: 10.1007/s11067-007-9027-6, Networks and Spatial Economics, 7(4), 315-331 (2007)

Phatarapon V., Pongtep P., Suparda K y Nuchananporn P., A comparison of Spatial Interpolation Methods for predicting concentrations of Particle Pollution (PM10), International Journal of Chemical, Environmental \& Biological Sciences (IJCEBS), 3(4), 302-306 (2015)

Reggiani, A., Bucci, P. y Russo, G., Accessibility and Network structures in the German Commuting, Network and Spatial Economics, 11(4), pp. 621-641, disponible en: https://goo.gl/RzXkfc (2010)

Reggiani, A., Bucci, P., Russo., G, Haas, A. y Nijkamp, P., Regional labour markets and job accessibility in city network systems in Germany, doi:10.1016/j.jtrangeo.2010.05.008, Journal of Transport Geography, 19, 528-536 (2011)

Rietveld, P. y Nijkamp P., Transport and regional development. In: J. Polak and A. Heertje, Editors, European Transport Economics, European Conference of Ministers of Transport (ECMT), Blackwell Publishers, Oxford (1993)

Shen, G., Measuring Accessibility of Housing to Public-community Facilities Using Geographical Information Systems, doi: 10.1111/1467-940X.00056, Review of Urban \& Regional Development Studies 14(3), 235-255 (2002)

Tanser, F., Gijsbertsen, B. y Herbst, K., Modelling and understanding primary health care accessibility and utilization in rural South Africa: an exploration using a geographical information system, doi: 10.1016/j.socscimed.2006.01.015, Social Science \& Medicine, 63(3),691-705 (2006)

Tsou, K., Hung, Y. y Chang, Y., An accessibility-based integrated measure of relative spatial equity in urban public facilities, Cities, 22(6), 424-435 (2005)

Vega, A., A multi-modal approach to sustainable accessibility in Galway, doi: 10.1080/20429843.2011.9727923, Regional Insights, 2(2), 15-17 (2011)

Vickerman, R., Spiekermann, K. y Wegener, M., Accessibility and economic development in Europe, doi:10.1080/00343409950118878, Regional Studies, 33 (1), 1-15 (1999)

Waddell, P., UrbanSim: Modeling urban development for land use, transportation, and environmental planning, doi:10.1080/01944360208976274, Journal of the American Planning Association, 68(3), 297-314 (2002)

Xia, J., Wright, J. y Adams, C., Five large Chinese biomedical bibliographic databases: accessibility and coverage, doi: 10.1111/j.1471-1842.2007.00734.x., Health Information \& Libraries Journal, 25(1), 55-61, (2008)

Younes, C., Escobar, D. y Holguín, J., Equidad, Accesibilidad y Transporte. Aplicación explicativa mediante un Análisis de Accesibilidad al Sector Universitario de Manizales (Colombia), doi:10.4067/S071807642016000300010. Inf. Tecnol. [online]. 27(3), 107-118 (2016)

Zhang, H. y Gao, Z., Bilevel programming model and solution method for mixed transportation network design problem, doi: 10.1007/s11424-009-9177-3, Journal of Systems Science and Complexity, 22 (3), 446-459 (2009) 